\title{
Percepção dos pacientes sobre a internação hospitalar em diferentes clínicas: uma revisão integrativa
}

\section{Perception of patients about hospitalization in different clinics: an integrative review \\ Percepción de los pacientes acerca de la hospitalización en diferentes clínicas: una revisión integradora}

Recebido: 28/02/2019

Aprovado: $27 / 07 / 2020$

Publicado: 07/03/2021

\author{
Patrícia Nunes da Silva ${ }^{1}$ \\ Lúcia Aparecida Ferreira²
}

Esta é uma revisão integrativa que considerou o período de 2015 a 2019, e teve como objetivo avaliar e sintetizar evidências disponíveis acerca da percepção dos pacientes sobre a internação hospitalar. A pesquisa foi realizada nas bases de dados: PubMed; CINAHL; Scopus; LILACS; BDENF, pelas combinações entre três descritores: Paciente, Percepção e Hospitalização. Encontraram-se 465 artigos, dos quais 11 artigos atenderam aos critérios do estudo. Todos os artigos apresentavam nível de evidencia 4, das quais emergiram duas categorias: Sentimentos vivenciados durante a internação e a percepção sobre o ambiente hospitalar; e Cuidado oferecido pelos profissionais de enfermagem durante a hospitalização. Quatro estudos eram brasileiros e sete eram estrangeiros. Os estudos revelaram a importância de o profissional de saúde estar preparado para o desenvolvimento de um conjunto de habilidades práticas e humanas, considerando a singularidade de cada paciente. Também verificou-se que, apesar dos avanços tecnológicos e da implementação das leis voltadas para a assistência humanizada, os profissionais de saúde possuem dificuldades para promover um cuidado integral e humanizado, garantindo qualidade na assistência.

Descritores: Hospitalização; Pacientes; Pacientes internados; Percepção; Pesquisa sobre serviços de saúde.

This is an integrative review that considered the period from 2015 to 2019, and aimed to evaluate and synthesize available evidence on the perception of patients about hospitalization. The research was carried out in the databases: PubMed; CINAHL; Scopus; LILACS; BDENF, with the combinations of three descriptors: Paciente, Percepção and Hospitalização (Patient, Perception and Hospitalization). 465 articles were found, of which 11 articles met the study criteria. All articles had evidence level 4, from which two categories emerged: Feelings experienced during hospitalization and the perception of the hospital environment; and Care offered by nursing professionals during hospitalization. Four studies were from Brazil and seven were from other countries. The studies revealed the importance of the health professional being prepared for the development of a set of practical and human skills, considering the uniqueness of each patient. It was also found that, despite technological advances and implementation of laws aimed at humanized care, health professionals have difficulties to promote comprehensive and humanized care, thus guaranteeing quality care.

Descriptors: Hospitalization; Patients; Impatients; Perception; Health services research.

Esta es una revisión integradora que consideró el período comprendido entre 2015 y 2019, cuyo objetivo fue evaluar y sintetizar evidencias disponibles de la percepción de los pacientes acerca de la hospitalización. La búsqueda se realizó en las bases de datos: PubMed; CINAHL; Scopus; LILACS; BDENF, por combinaciones de tres descriptores: Paciente, Percepção e Hospitalização (Paciente, Percepción y Hospitalización). Se encontraron 465 artículos, de los cuales 11 cumplieron los criterios. Todos los artículos presentaron un nivel de evidencia 4, y surgieron dos categorías: Sentimientos experimentados durante la hospitalización y percepción del entorno hospitalario; y Cuidado ofrecido por los profesionales de enfermería durante la hospitalización. Cuatro estudios eran brasileños y siete eran extranjeros. Los estudios revelaron la importancia de que el profesional de salud esté preparado para el desarrollo de un conjunto de habilidades prácticas y humanas, considerando la singularidad de cada paciente. Asimismo, se constató que a pesar de los avances tecnológicos y de la implementación de leyes orientadas a la atención humanizada, los profesionales de la salud tienen dificultades para promover un cuidado integral y humanizado, garantizando una atención de calidad.

Descriptores: Hospitalización; Pacientes; Pacientes internos; Percepción; Investigación sobre servicios de salud.

1. Enfermeira. Especialista em Saúde do Adulto. Uberaba, MG, Brasil. ORCID: 0000-0001-9321-577X E-mail: patricia.n18@hotmail.com 2. Enfermeira. Especialista em Educação Profissional em Saúde. Mestre em Enfermagem Psiquiátrica. Doutora em Enfermagem. Professora Associada e Coordenadora do curso de Enfermagem da Universidade Federal do Triângulo Mineiro, Uberaba, MG, Brasil. ORCID: 0000-0001-6469-5444 E-mail: lap2ferreira@yahoo.com.br 


\section{INTRODUÇÃO}

A

necessidade de hospitalização, resultante do agravamento clínico pode-se caracterizar como um processo traumático e estressor, uma vez que o ambiente hospitalar possui regras e normas de funcionamento, culminando em condições restritivas ${ }^{1}$. Esta situação torna-se fonte de mudanças comportamentais, podendo gerar desconforto, associando-se à configuração ambiental aversiva, o que pode dificultar o processo de adaptação necessário para enfrentamento da doença².

O sujeito hospitalizado encontra-se em uma condição na qual sua saúde está ameaçada ou prejudicada, e necessita abandonar seu cotidiano para assumir uma nova conjuntura. Neste período, ocorrem rupturas do sujeito com seu dia-a-dia, distanciando-se do convívio familiar e de amigos, afastando-se do trabalho e das atividades de lazer, além da perda de autonomia, autoestima e reconhecimento social ${ }^{3,4}$.

Quando o indivíduo passa por internação hospitalar, sabe-se que não é apenas a sua dimensão física que está sendo acometida pela enfermidade, mas o sujeito em si, em sua totalidade. Cada sujeito possui uma história, uma individualidade, carrega consigo particularidades específicas e singulares, as quais devem ser reconhecidas ${ }^{5}$ e consideradas como dimensões importantes para o cuidado.

O fortalecimento da autonomia e a compreensão da condição clínica contribuem para a quebra de estigmas e preconceitos e, consequentemente, permitem fluidez no enfrentamento da doença e superação das fragilidades e dificuldades no ambiente hospitalar ${ }^{6}$.

O profissional de saúde que acompanha o paciente durante sua internação precisa levar em consideração o indivíduo em sua totalidade ${ }^{7}$. É crucial que os profissionais prestem um cuidado integral e comprometido desde a entrada do indivíduo no hospital. Uma comunicação efetiva e o estabelecimento de vínculos possibilitam uma análise detalhada das percepções e reações vivenciadas pelo paciente hospitalizado ${ }^{3,8}$.

A relação terapêutica, baseada na compreensão do indivíduo em sua totalidade, contribui com desenvolvimento de intervenções individuais e focadas melhorando a experiência da hospitalização ${ }^{8}$, uma vez que instiga olhar crítico e interrogador capaz de modificar a assistência prestada ao indivíduo ${ }^{9}$. Diante disto, chama-se atenção para a humanização enquanto nova postura e atitude no contexto do trabalho em saúde, a qual precisa envolver desde as ações profissionais até a organização dos serviços e gestão.

A humanização é um processo amplo, e revela-se como uma ferramenta fundamental no cuidado com o paciente hospitalizado, não devendo limitar-se apenas às etapas procedimentais, mas precisa fundamentar-se em um processo mais amplo e interativo que envolva usuário, familiares e equipe multiprofissional ${ }^{10}$, promovendo um ambiente hospitalar respeitoso que preserve a privacidade e a dignidade de cada paciente ${ }^{11}$.

Apesar das novas políticas públicas de saúde destacarem a atenção integral do sujeito, houve desenvolvimento científico, tecnológico e superespecialização na área da saúde que trouxeram benefícios incontestáveis e melhoria para o cuidado em saúde; porém, notou-se que a assistência foi se distanciando da singularidade do sujeito. Os profissionais tornaram-se excelentes técnicos, detentores de saber científico e conhecedores de procedimentos, muitas vezes, de alta complexidade. Entretanto, afastaram-se dos aspectos humanos e da dimensão não palpável do indivíduo, o que gerou um distanciamento cada vez maior dos sujeitos que eles assistem $^{5}$. Constata-se a necessidade de redirecionar o olhar para o indivíduo, humanizando o cuidado em saúde ${ }^{12}$, tendo como guia para a assistência o ser humano em sua totalidade, e não a soma de seus fragmentos e partes desconexas.

Frente a esta realidade, é relevante pesquisar sobre os cuidados e intervenções a partir da percepção do paciente, compreendendo o universo representacional dos indivíduos que vivenciam a internação hospitalar. Neste contexto, a produção de estudos e conteúdos nesta área podem contribuir para que os profissionais reflitam na questão da humanização em suas 
atuações, na importância de compreender o paciente como um ser complexo, singular, capaz de modificar-se, dependendo das relações e condições ambientais que interagem.

Trazer para o centro das discussões a percepção do paciente sobre a hospitalização pode impactar diretamente no seu próprio cuidado e representar um passo decisivo para que as atitudes dos profissionais sejam revisadas. Nesta perspectiva, emergiu o seguinte questionamento: Qual a percepção dos indivíduos em relação à sua internação hospitalar? Assim, este estudo tem como objetivo avaliar e sintetizar evidências disponíveis acerca da percepção dos pacientes sobre a internação hospitalar.

\section{MÉTODO}

Trata-se de um estudo de revisão integrativa (RI) definida como um método de pesquisa que visa elencar o maior número de estudos acerca de uma temática, que resulta em um estudo fundamentado de suporte ao conhecimento e a prática clínica13.

Foram utilizados seis passos descritos para a construção do estudo: estabelecimento da hipótese ou questão de pesquisa; amostragem ou busca na literatura; categorização dos estudos; avaliação dos estudos incluídos na revisão; interpretação dos resultados e síntese do conhecimento ou apresentação da revisão ${ }^{13}$.

0 intervalo de tempo para seleção dos artigos foi de cinco anos (2015-2019). A coleta dos artigos ocorreu no mês de agosto de 2019. A pesquisa foi realizada em seis bases de dados eletrônicos, com acesso on-line: Cumulative Index to Nursing and Allied Health Literatue (CINAHL); PubMed/Medline (National Library of Medicine and National Institutes of Health/Medical Literature Analysis and Retrieval System Online); Scopus; Literatura LatinoAmericana e do Caribe em Ciências da Saúde (LILACS), Medical Literature Analysis and Retrieval System Online (MEDLINE) e Base de Dados de Enfermagem (BDENF). 0 acesso às bases de dados deu-se da seguinte maneira: para acessar CINAHL e Scopus, utilizou-se o Portal de Periódicos da Coordenação de Aperfeiçoamento de Pessoal de Nível Superior (CAPES); para acessar PubMed utilizou-se o portal PubMed; para a LILACS, BDENF e MEDLINE o acesso foi via Biblioteca Virtual de Saúde (BVS).

Foram utilizadas combinações entre três descritores (pacientes, percepção e hospitalização) em português e seus correspondentes em inglês (patients, perception e hospitalization), via Descritores em Ciências da Saúde (DeCS). Utilizaram-se combinação dos descritores com os operadores boolianos ("and", "or", "not" ou "and not"), respeitando as distinções entre as bases de dados.

Estabeleceu-se como critérios de inclusão artigos completos publicados em periódicos científicos disponíveis nas bases de dados online selecionadas para o estudo nos últimos cinco anos (2015-2019), nos idiomas português, inglês e espanhol, e que tinham como temática a percepção dos pacientes acerca da hospitalização.

Optou-se por analisar somente os estudos em que a amostra era constituída por pacientes adultos e abrangendo diferentes clínicas de internação hospitalar. Foram excluídos artigos incompletos, teses, dissertações, revisões, resumos em anais de eventos, resumos expandidos, cartas, editoriais e estudos realizados em cenários não hospitalares. Também foram excluídos artigos que abordavam a perspectiva de crianças, adolescentes e idosos internados, familiares e/ou acompanhantes.

A avaliação e seleção dos estudos que integram o corpus foi efetivada com base na leitura dos artigos para averiguar se, de fato, respondiam aos objetivos.

A primeira etapa de análise dos estudos identificados ocorreu por meio da leitura dos títulos e resumos das referências identificadas nas bases de dados. Notou-se a ocorrência de artigos duplicados, os quais foram excluídos, respeitando a base de dados de maior indexação. Logo após, foi realizada a leitura flutuante da seleção primária dos artigos, na qual foi constatado que nem todos artigos contemplavam os critérios estabelecidos. 
Posteriormente, fez-se a exclusão deles, resultando na redução do número de artigos. Desse modo, procedeu-se com uma leitura sistematizada dos artigos que, finalmente, atendiam aos critérios de inclusão e que tratavam sobre percepção dos pacientes acerca da hospitalização. Finalmente, após definir a amostra, os artigos que compuseram ao corpus do estudo foram organizados em arquivos físicos e virtuais.

Os artigos foram analisados e classificados quanto ao nível de evidência, conforme o método utilizado. 0 nível 1 aponta evidências resultantes da meta-análise de múltiplos estudos clínicos controlados e randomizados; nível 2 contempla evidências obtidas em estudos individuais com delineamento experimental; nível 3 são evidências de estudos quaseexperimentais; nível 4 consiste em evidências de estudos descritivos (não-experimentais) ou com abordagem qualitativa; nível 5 considera evidências provenientes de relatos de caso ou de experiência; nível 6 são evidências baseadas em opiniões de especialistas ${ }^{14}$.

A apresentação desta revisão foi realizada com a disposição dos resultados obtidos em quadros e em análise narrativa para facilitar a identificação e compreensão dos dados. Foi criado quadros para a coleta das informações contendo os seguintes itens: autores, nível de evidência, data de publicação, base de dados, periódicos, objetivo e local de pesquisa. Os dados foram descritos utilizando-se frequência absoluta (n) e percentual (\%). Após análise e interpretação dos resultados obtidos, emergiram categorias que foram discutidas com produções relacionadas.

\section{RESULTADOS}

Nas proposições do estudo, considerou-se 11 artigos, que constam na Figura 1.

Figura 1. Fluxograma de seleção dos estudos primários, Uberaba, Minas Gerais, Brasil 2020.

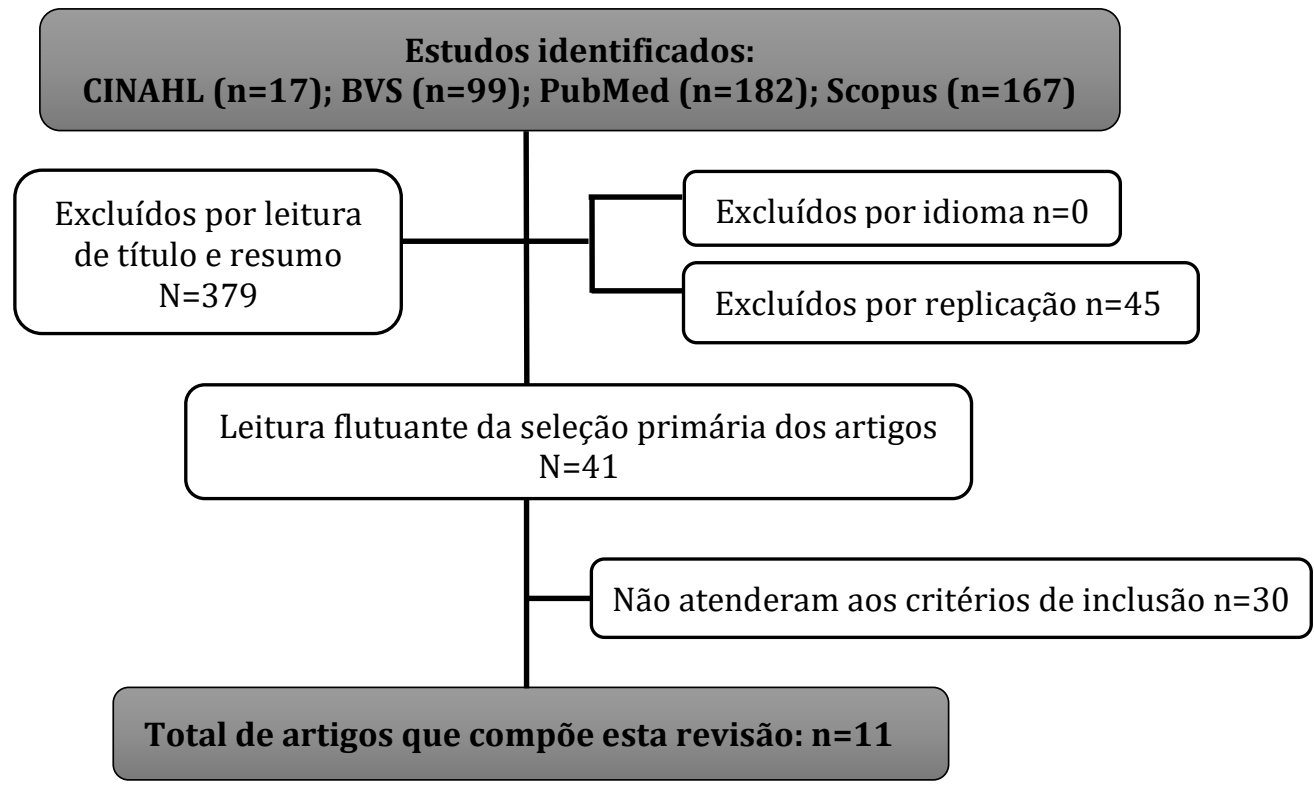

Referente ao ano de publicação, constatou-se que o ano 2016 apresentou maior número de artigos selecionados, correspondendo a 36,4\% $(n=4)$ das publicações, seguido dos anos de 2015, 2017 e 2018, com 18,2\% cada (n=2). 0 ano de 2019 corresponde a 9,1\% (n=1) das publicações (Quadro 1).

Em virtude das características específicas para observar e descrever a percepção dos pacientes, todos os estudos, $100 \%(\mathrm{n}=11)$, foram classificados com nível de evidência 4 , (Quadro 1).

Foi resgatado o maior número de publicações na base de dados Scopus 27,3\% (n=3). As bases Medline, BDENF e LILACS apresentaram o mesmo número de publicações resgatadas, 
sendo 18,2\% (n=2). Já as bases de dados PubMed e CINAHL foram resgatadas apenas uma publicação cada 9,1\% (n=1) (Quadro 1).

Os onze estudos incluídos na revisão foram publicados nos seguintes períodicos, a saber: Journal of Critical care $(\mathrm{n}=1)$, Medical Principles and Practice $(\mathrm{n}=1)$, Archives of Psychiatric Nursing $(\mathrm{n}=1)$, CuidArte Enfermagem $(\mathrm{n}=1)$, European Journal of Oncology Nursing $(n=1)$, Hispanic Health Care International $(\mathrm{n}=1)$, Revista Eletrônica de Enfermagem $(\mathrm{n}=1)$, Journal of Research: Fundamental Care Online $(\mathrm{n}=1)$, Revista de Enfermagem da UFSM ( $\mathrm{n}=1)$, European journal of Cancer Care $(\mathrm{n}=1)$, International Journal for Quality in Health Care $(\mathrm{n}=1)$ (Quadro 1).

Quanto aos países que mais publicaram sobre o tema, destaca-se o Brasil com o maior número do total de publicações selecionadas, sendo 36,4\% (n=4). Arábia Saudita, China, Estados Unidos, Índia, México, Jordânia, Portugal e Polônia tiveram apenas uma publicação cada, o que equivale a 9,1\%. Ressalta-se que um único estudo foi realizado em quatro países, nominalmente Chipre, Finlândia, Grécia e Suécia, caracterizando-se na amostra com 9,1\% (n=1) (Quadro 1).

Quadro 1. Artigos selecionados segundo autores, ano, objetivo, base de dados, periódicos e país de estudo. Uberaba, Minas Gerais, Brasil, 2020.

\begin{tabular}{|c|c|c|c|c|c|}
\hline Autor/Ano & $\begin{array}{l}\text { Nível de } \\
\text { Evidência }\end{array}$ & Objetivo & $\begin{array}{l}\text { Bases } \\
\text { de } \\
\text { Dados }\end{array}$ & Periódico & $\begin{array}{l}\text { País de } \\
\text { Estudo }\end{array}$ \\
\hline $\begin{array}{l}\text { Alasad JA, et } \\
\text { al.15 }(2015)\end{array}$ & 4 & $\begin{array}{l}\text { Descrever a experiência dos pacientes da Jordânia } \\
\text { durante sua permanência em tratamento intensivo. }\end{array}$ & MEDLINE & $\begin{array}{l}\text { Journal of } \\
\text { Critical care }\end{array}$ & Jordânia \\
\hline $\begin{array}{l}\text { Al-Monami } \\
\text { MM16 } \\
(2016)\end{array}$ & 4 & $\begin{array}{l}\text { Investigar a satisfação dos pacientes com os } \\
\text { cuidados de enfermagem medindo a diferença } \\
\text { entre as expectativas dos pacientes e percepços } \\
\text { dos reais cuidados prestados. }\end{array}$ & Scopus & $\begin{array}{l}\text { Medical } \\
\text { Principles } \\
\text { and Practice }\end{array}$ & $\begin{array}{l}\text { Arábia } \\
\text { Saudita }\end{array}$ \\
\hline $\begin{array}{l}\text { Bandurska E, } \\
\text { et al. }{ }^{17} \\
(2016)\end{array}$ & 4 & $\begin{array}{l}\text { Avaliar a qualidade da assistência de enfermagem } \\
\text { em enfermarias psiquiátricas sob a perspectiva do } \\
\text { paciente. }\end{array}$ & PubMed & $\begin{array}{l}\text { Archives of } \\
\text { Psychiatric } \\
\text { Nursing, }\end{array}$ & Polônia \\
\hline $\begin{array}{l}\text { Beccaria LM, } \\
\text { et al. } 18 \\
(2018)\end{array}$ & 4 & $\begin{array}{l}\text { Verificar a percepção do paciente e seu familiar } \\
\text { quanto à experiência de internação em unidade } \\
\text { coronária após cirurgia cardíaca. }\end{array}$ & BDENF & $\begin{array}{l}\text { CuidArte, } \\
\text { Enfermagem }\end{array}$ & Brasil \\
\hline $\begin{array}{l}\text { Garza- } \\
\text { Hernández R, } \\
\text { et al.19 } \\
\text { (2019) }\end{array}$ & 4 & $\begin{array}{l}\text { Descrever, em geral e por categorias, a percepção } \\
\text { dos pacientes sobre comportamentos de cuidados } \\
\text { humanizados que foram fornecidos pela equipe de } \\
\text { enfermagem durante a internação, em serviços } \\
\text { cirúrgicos, em um hospital de cuidados. }\end{array}$ & Scopus & $\begin{array}{l}\text { Hispanic } \\
\text { Health Care } \\
\text { International }\end{array}$ & México \\
\hline $\begin{array}{l}\text { Kullberg A, et } \\
\text { al.20 } \\
(2015)\end{array}$ & 4 & $\begin{array}{l}\text { Investigar a percepção dos pacientes sobre troca de } \\
\text { informações e suas associações com a satisfação do } \\
\text { paciente, participação e segurança na internação } \\
\text { em alas de oncologia. }\end{array}$ & Scopus & $\begin{array}{l}\text { European } \\
\text { Journal of } \\
\text { Oncology } \\
\text { Nursing }\end{array}$ & Suécia \\
\hline $\begin{array}{l}\text { Martins PF, } \\
\text { Perroca MG } \\
\quad \text { (2017) }\end{array}$ & 4 & $\begin{array}{l}\text { Examinar a percepção e o nível de satisfação de } \\
\text { pacientes e acompanhantes quanto ao atendimento } \\
\text { das necessidades de cuidados durante o período de } \\
\text { hospitalização. }\end{array}$ & LILACS & $\begin{array}{l}\text { Revista } \\
\text { Eletrônica de } \\
\text { Enfermagem }\end{array}$ & Brasil \\
\hline $\begin{array}{l}\text { Piexak DR, et } \\
\text { al. }(2016)^{3}\end{array}$ & 4 & $\begin{array}{l}\text { Compreender o significado do cuidado de } \\
\text { enfermagem em uma unidade de internação } \\
\text { cirúrgica na percepção dos pacientes. }\end{array}$ & LILACS & $\begin{array}{l}\text { Journal of } \\
\text { Research: } \\
\text { Fundamental } \\
\text { Care Online. }\end{array}$ & Brasil \\
\hline $\begin{array}{l}\text { Silva N, } \\
\text { Gabatz RI, } \\
\text { Lemes RA } \\
\text { (2016) }\end{array}$ & 4 & $\begin{array}{l}\text { Identificar a percepção do paciente traumatológico } \\
\text { acerca da assistência prestada. }\end{array}$ & BDENF & $\begin{array}{l}\text { Revista de } \\
\text { Enfermagem } \\
\text { da UFSM }\end{array}$ & Brasil \\
\hline $\begin{array}{l}\text { Suhonen R, et } \\
\text { al.23 } \\
(2018)\end{array}$ & 4 & $\begin{array}{l}\text { Descrever as percepções dos pacientes com câncer } \\
\text { de atendimento individualizado em quatro países } \\
\text { europeus e comparar essas percepções usando as } \\
\text { características sociodemográficas dos pacientes e } \\
\text { Escala de Atenção Individualizada. }\end{array}$ & MEDLINE & $\begin{array}{l}\text { European } \\
\text { Journal of } \\
\text { Cancer Care }\end{array}$ & $\begin{array}{l}\text { Chipre } \\
\text { Finlândia } \\
\text { Grécia } \\
\text { Suécia }\end{array}$ \\
\hline $\begin{array}{l}\text { Yan J, et al. }{ }^{24} \\
\quad(2017)\end{array}$ & 4 & $\begin{array}{l}\text { Identificar a frequência de eventos indesejáveis } \\
\text { relatados pelos pacientes durante a hospitalização. }\end{array}$ & CINAHL & $\begin{array}{l}\text { International } \\
\text { Journal for } \\
\text { Quality in } \\
\text { Health Care }\end{array}$ & China \\
\hline
\end{tabular}


Em relação à caracterização, 18,2\% (n=2) dos estudos focalizaram exclusivamente cuidados intensivos, 18,2\% (n=2); departamento cirúrgico, 18,2\% (n=2) envolviam apenas clínicas médica e cirúrgica, 9,1\% $(n=1)$ em departamentos variados, 9,1\% $(n=1)$ oncológico, $9,1 \%(n=1)$ psiquiátricos e $9,1 \%(n=1)$ o departamento de traumatologia.

No que se refere ao nível de evidencia, todos são de nível 4, mostrando importante lacuna no tema para pesquisas de perspectivas de diferentes desenhos, como de 1 a 3, a saber: nível 1 - meta-análise de múltiplos estudos clínicos controlados e randomizados; nível 2 - estudos individuais com delineamento experimental; nível 3 - estudos quase-experimentais ${ }^{14}$.

A categorização dos dados permitiu emergir duas categorias a serem discutidas: Sentimentos vivenciados durante a internação e a percepção sobre o ambiente hospitalar; e Cuidado oferecido pelos profissionais de enfermagem durante a hospitalização.

\section{DISCUSSÃO}

\section{Sentimentos vivenciados durante a internação e a percepção sobre o ambiente hospitalar}

0 período de internação representa uma condição complexa que interfere diretamente em todos os aspectos do indivíduo, que assume uma condição passiva em relação ao seu cuidado ${ }^{18}$. Os sentimentos vivenciados neste período são singulares e distintos, muitas vezes influenciados por: valores, crenças, expectativas, ambiente, experiências anteriores e condição física e psicossocial ${ }^{16,23}$.

Em estudo realizado na região central do Rio Grande do Sul, Brasil, apurou-se que o cuidado deve compreender aspectos físicos, emocionais, sociais e espirituais do paciente, sendo fundamental manter uma escuta atenta e sensível interligada à comunicação, respeitando as particularidades de cada um para o alcance da efetividade. Essa compreensão se deve ao fato dos sentimentos se apresentarem de maneira distinta e singular ${ }^{3}$.

A alteração do cotidiano e o enfrentamento de dificuldades expõe os pacientes ao medo, angústia, ansiedade e preocupação, contribuindo para que esse período se torne estressante. Outros pacientes podem vivenciar esse momento como uma experiência necessária, apegandose em sentimentos de fé e esperança. Ambas perspectivas devem ser reconhecidas e consideradas como determinantes no enfrentamento da doença e da hospitalização ${ }^{3}$.

Dos artigos que se enquadram na discussão acerca dos sentimentos vivenciados durante a internação, três destacavam a necessidade cirúrgica como um contribuinte para aumentar o estresse e a ansiedade nos pacientes. Esses transtornos são potencializados pelo medo de modificar rotina, hábitos e costumes, afastando-os do convívio social, familiar e do trabalho. Os pacientes podem apresentar dificuldades em aceitar a internação cirúrgica devido à baixa perspectiva gerada pela ausência de informações sobre sua condição e sobre os procedimentos ${ }^{3,18,22}$.

O ambiente hospitalar pode acarretar situações de estresse, uma vez que impõe condições restritivas e mudanças repentinas que atrapalham o processo de adaptação dos pacientes ${ }^{24}$. Dentre as dificuldades vivenciadas durante a internação, destaca-se o distanciamento do convívio familiar causando uma ruptura da interdependência afetiva e emocional influenciando diretamente no processo saúde-doença ${ }^{18}$.

Cabe aos profissionais implementarem medidas para superar as dificuldades e restrições, além de orientar os familiares quanto à condição de saúde e a rotina hospitalar, promovendo um ambiente de interação que garanta a participação consciente e maior bem-estar durante evolução do paciente ${ }^{22}$.

Alguns estudos correlacionam outros fatores estressores no ambiente hospitalar, como: a presença de ruídos e luzes excessivas provocados pela equipe de saúde e por equipamentos. A equipe deve estar atenta, minimizando os ruídos provocados que influenciam negativamente no processo de hospitalização dificultando o repouso, essencial para a reabilitação ${ }^{18,22}$. 


\section{Cuidado oferecido pelos profissionais de enfermagem durante a hospitalização}

A internação é compreendida de diferentes formas entre as pessoas, variando de acordo com a experiência individual. É necessário ampliar a visão mecanicista e procedimental, tendo a integralidade e a humanização como principais norteadores, além de compreender a complexidade que envolve o processo de cuidar de seres humanos em sua singularidade ${ }^{21}$ garantindo uma assistência de qualidade.

Um estudo que avaliou a percepção dos pacientes cirúrgicos sobre a assistência de enfermagem humanizada corrobora a necessidade de repensar a assistência sob a lógica da integralidade e da humanização, o que permitiu identificar que as percepções dos pacientes alcançaram pontuações favoráveis quando a equipe de enfermagem fornecia cuidados pautados na humanização ${ }^{19}$. Comportamentos relacionados ao respeito, a dignidade e a privacidade sem distinção de crenças e valores, reconhecimento das necessidades individuais, valorização dos sentimentos, comunicação efetiva e atitudes que transmite tranquilidade e segurança durante a hospitalização foram determinantes ${ }^{19}$.

0 cuidado centrado na pessoa é impulsionado por necessidades e preferências dos pacientes que promove satisfação, participação e segurança. Desse modo, as ações de saúde devem incentivar o envolvimento do paciente no seu próprio cuidado, visando fortalecer o seu papel e autonomia ${ }^{21}$. 0 cuidado centralizado facilita a detecção e prevenção de eventos adversos, uma vez que os pacientes participam no processo dos seus cuidados em saúde, sendo capazes de fornecer informações mais detalhadas do seu estado, contribuindo com sua segurança no ambiente hospitalar ${ }^{24}$.

Os pacientes não esperam receber, somente, cuidados de natureza puramente físicas, para solucionar diagnósticos e tratar sintomas. Esperam receber apoio psicológico, uma boa comunicação, disponibilidade da equipe para esclarecer dúvidas e manter relações de parcerias despertando sensações de confiança e de segurança. A assistência, pautada nesses aspectos, provoca impacto positivo sobre o estado de sua saúde, sendo necessário considerá-las e avaliálas para elaboração de estratégias que diligenciam o aperfeiçoamento o cuidado ${ }^{17}$.

Outro estudo que avaliou a percepção de pacientes internados com câncer sobre os cuidados recebidos pela equipe de enfermagem enfatiza que a individualidade e a escolha de cada paciente devem ser consideradas ${ }^{23}$. Percebeu-se que a implementação de cuidados individualizados no contexto do tratamento do câncer foi moderada, não suprindo as expectativas que eram significativamente maiores. Este aspecto negativo está relacionado com uma possível deficiência na formação complementar especializada dos profissionais e pela sobrecarga de tarefas que impossibilita uma comunicação efetiva e estabelecimento de vínculo, necessários para implementar um cuidado individualizado ${ }^{23}$.

Os pacientes encontram-se, muitas vezes, fisicamente ou psicologicamente vulneráveis devido ao estado de saúde e a condição da qual estão submetidos no ambiente hospitalar. Somado à essa condição, a falta de comunicação verbal ou não verbal pode impactar negativamente a satisfação do paciente com a assistência recebida, uma vez que experiências inesperadas podem ser graves e traumáticas. A troca de informações claras buscam otimizar o atendimento e contribuir para que os pacientes sintam-se mais seguros durante o período de hospitalização ${ }^{15,20,24}$.

Nesse sentido, a comunicação efetiva é primordial para se alcançar uma assistência que legitima os pacientes como verdadeiros parceiros no cuidado. A troca de informações eficazes é crucial tanto para o paciente quanto para o profissional que o assiste, não podendo ser superestimada $^{20}$. Os pacientes que reconhecem suas reais necessidades são capazes de participarem ativamente no planejamento e na tomada de decisões sobre seus cuidados, garantindo segurança e satisfação ${ }^{21}$. Para o profissional, a troca de informações proporciona possibilidades para avaliar e educar pacientes e familiares, executar o gerenciamento de sintomas e coordenar os cuidados fundamentados nas reais necessidades dos pacientes ${ }^{20}$. 
No que se refere às inter-relações estabelecidas no período de internação, a equipe de enfermagem, por ter maior contato direto durante a internação, tende a ser vista pelos pacientes como uma figura protetora. Diante disso, a equipe de enfermagem tem papel fundamental na construção de vínculos para estabelecer cuidado a partir das necessidades expressas pelos pacientes, minimizando os efeitos negativos da internação ${ }^{16,18}$.

\section{CONCLUSÃo}

A percepção do paciente sobre a internação é concernente com o estado emocional após experiências positivas e negativas envolvendo o ambiente hospitalar, a equipe e o processo terapêutico. Diversos sentimentos podem surgir durante a hospitalização, o que interfere diretamente no processo saúde-doença. 0 cuidado integral comprometido com estabelecimento de vínculos para atender as reais necessidades expressas pelos pacientes é imprescindível para a segurança, participação e satisfação dos pacientes.

Os estudos revelaram a importância de o profissional de saúde estar preparado para o desenvolvimento de um conjunto de habilidades práticas e humanas, considerando a singularidade de cada paciente. Percebe-se a relevância da construção de um cuidado que considere as necessidades expressas para a prática clínica, amenizando possíveis dificuldades e sofrimentos vivenciados pelos pacientes no período de hospitalização.

Os artigos avaliados permitiram evidenciar que, apesar dos avanços tecnológicos e da implementação das leis voltadas para a assistência humanizada, os profissionais de saúde possuem dificuldades para promover um cuidado integral e humanizado, garantindo qualidade na assistência. Nesse sentido, percebeu-se que as implicações do cuidado baseado nas percepções e necessidades do paciente são grandes e muito há que ser feito para que se alcance uma assistência humanizada que respeite a singularidade dos sujeitos assistidos durante a hospitalização.

As limitações do estudo relacionam-se ao método utilizado, ao recorte temporal e a escassez de estudos primários com melhores níveis de evidência, revelando-se como uma lacuna na produção científica. A amplitude da temática em diferentes clínicas, também se torna um fator limitante, uma vez que o cenário e a condição clínica influência de diferentes formas. Por outro lado, foi observado a mesma necessidade em valorizar a subjetividade do sujeito frente à um cuidado integral em ambos os cenários.

Além disso, os resultados possuem limitações em relação ao nível de evidência dos estudos. Apesar destas limitações, acredita-se que o estudo pode contribuir nas discussões sobre a temática, no delineamento de modelos de intervenção, bem como na transformação da prática, minimizando os impactos na assistência à saúde.

Portanto, sugere-se mais pesquisas científicas que envolvam estratégias para implementação de assistência que reconheça todas as dimensões inerentes ao paciente, respeitando a singularidade e necessidades, com vistas a melhorias no cuidado.

\section{REFERÊNCIAS}

1. Ribeiro LC, Luna VL, Medeiros KT. Estratégias de enfrentamento das doenças por idosas hospitalizadas. Psico-USF [Internet]. 2018 [citado em 10 ago 2019]; 23(3):473-82. Disponível em: http://www.scielo.br/pdf/pusf/v23n3/2175-3563-pusf-23-03-473.pdf. $\quad$ DOI: https://doi.org/10.1590/1413-82712018230307

2. Campos CG, Mantovani MD, Nascimento ME, Cassi CC. Representações sociais sobre o adoecimento de pessoas com doença renal crônica. Rev Gaúcha Enferm. [Internet]. 2015 [citado em 06 ago 2019]; 36(2):106-12. Disponível em: https://seer.ufrgs.br/RevistaGauchadeEnfermagem/article/view/48183/34199. DOI: http://dx.doi.org/10.1590/1983-1447.2015.02.48183

3. Piexak DR, Ferreira CL, Terra MG, Backes DS, Barlem JG, Ilha S. Cuidado de enfermagem em unidade de internação cirúrgica: percepção dos pacientes. Rev Pesqui. (Univ Fed Estado Rio J., 
Online) [Internet]. 2016 [citado em 8 dez 2019]; 8(1):3624-32. Disponível em: http://www.seer.unirio.br/index.php/cuidadofundamental/article/view/3596/pdf_1765

4. Reis CG, da Rosa Olesiak L, Quintana AM, Farias CP. Repercussões profissionais e cotidianas do adoecimento em pacientes do sexo masculino com câncer avançado. Psicol Pesq. [Internet]; 2018 [citado em 05 ago 2019]; 12(1):1-11. Disponível em: http://pepsic.bvsalud.org/pdf/psipesq/v12n1/07.pdf.

DOI: http://dx.doi.org/10.24879/2018001200100315

5. Backes DS, Lunardi VL, Lunardi Filho WD. A humanização hospitalar como expressão da ética. Rev Latinoam Enferm. [Internet]. 2006 [citado em 05 ago 2019]; 14(1):132-5. Disponível em: http://www.scielo.br/pdf/rlae/v14n1/v14n1a18.pdf. DOI: https://doi.org/10.1590/S010411692006000100018

6. Damion M, Moreira MC. Percepção do paciente sobre sua autonomia na Unidade de Terapia Intensiva. Contextos Clín. [Internet]. 2018 [citado em 05 ago 2019]; 11(3):386-96. Disponível em:

http://www.revistas.unisinos.br/index.php/contextosclinicos/article/view/ctc.2018.113.09/ 60746519

7. Carvalho DO, Santos NN, Silva AR, Carvalho GC. Percepção do profissional de enfermagem acerca do cuidado humanizado no ambiente hospitalar. Rev Interdisciplin. [Internet]. 2015 [citado em 05 ago 2019]; 8(3):61-74. Disponível em: https://revistainterdisciplinar.uninovafapi.edu.br/index.php/revinter/article/view/680/pdf_ 237

8. Liu T, Kiwak E, Tinetti M. Perceptions of hospital-dependent patients on their needs for hospitalization. J Hosp Med. [Internet]. 2017 [citado em 07 ago 2019]; 12(6):450-3. Disponível em: https://insights.ovid.com/johm/201706000/01445454-201706000-00010. DOI: https://doi.org/10.12788/jhm.2756

9. Oliveira JS, Suto CS, Silva RS. Tecnologias leves como práticas de enfermagem na atenção básica. Rev Saúde Com. [Internet]. 2016 [citado em 05 ago 2019]; 12(3):613-21. Disponível em: http://periodicos2.uesb.br/index.php/rsc/article/view/425/344

10. Ministério da Saúde (Br). Secretaria de Atenção à Saúde, Núcleo Técnico da Política Nacional de Humanização. HumanizaSUS: documento base para gestores e trabalhadores do SUS. Brasília, DF: Ministério da Saúde; 2010 [citado em 05 ago 2019]. Disponível em: http://bvsms.saude.gov.br/bvs/publicacoes/humanizasus_documento_gestores_trabalhadore s_sus.pdf

11. Hosseini FA, Momennasab M, Yektatalab S, Zareiyan A. Patients' perception of dignity in Iranian general hospital settings. Nurs Ethics [Internet]. 2019 [citado em 05 ago 2019]; 26(6):1777-90.

Disponível

em: https://journals.sagepub.com/doi/abs/10.1177/0969733018772078.

DOI: https://doi.org/10.1177/0969733018772078

12. Brito FM, Costa IC, Costa SF, Andrade CG, Santos KF, Francisco DP. Comunicação na iminência da morte: percepções e estratégia adotada para humanizar o cuidar em enfermagem. Esc Anna Nery Rev Enferm. [Internet]. 2014 [citado em 05 ago 2019]; 18(2):317-22. Disponível em: $\quad$ https://www.redalyc.org/pdf/1277/127730686020.pdf.

DOI: https://doi.org/10.5935/1414-8145.20140046

13. Mendes KD, Silveira RC, Galvão CM. Revisão integrativa: método de pesquisa para a incorporação de evidências na saúde e na enfermagem. Texto \& Contexto Enferm. [Internet]. 2008 [citado em 06 ago 2019]; 17(4):758-64. Disponível em: http://www.scielo.br/pdf/tce/v17n4/18.pdf. DOI: https://doi.org/10.1590/S010407072008000400018

14. Souza MT, Silva MD, Carvalho RD. Revisão integrativa: o que é e como fazer. Einstein (São Paulo) [Internet]. 2010 [citado em 26 jul 2020]; 8(1):102-6. Disponível em: https://www.scielo.br/pdf/eins/v8n1/pt_1679-4508-eins-8-1-0102 
15. Alasad JA, Tabar NA, Ahmad MM. Patients' experience of being in intensive care units. J Crit Care [Internet]. 2015 [citado em 18 dez 2019]; 30(4):859-e7. Disponível em: https://www.sciencedirect.com/science/article/abs/pii/S0883944115001306?via\%3Dihub. DOI: https://doi.org/10.1016/j.jcrc.2015.03.021

16. Al-Momani MM. Gap analysis between perceptions and expectations of medical-surgical patients in a public hospital in Saudi Arabia. Med Princ Pract. [Internet]. 2016 [citado em 18 dez 2019]; 25(1):79-84. Disponível em: https://www.karger.com/Article/Pdf/441000. DOI: https://doi.org/10.1159/000441000

17. Bandurska E, Zarzeczna-Baran M, Zielazny P. Wards in opinion of patients-a comparative study on the quality of nursing care. Arch Psychiatr Nurs. [Internet]. 2016 [citado em 18 dez 2019]; 30(6):685-91. Disponível em: https://www.sciencedirect.com/science/article/abs/pii/S0883941716300036. DOI: https://doi.org/10.1016/j.apnu.2016.03.006

18. Beccaria LM, Paleuco IC, Barbosa TP, Faria JI, Jacon JC. Internação em unidade coronária após cirurgia cardíaca: percepção do paciente e seu familiar. CuidArte Enferm. [Internet]. 2018 [citado em 18 dez 2019]; 12(1):92-7. Disponível em: http://www.webfipa.net/facfipa/ner/sumarios/cuidarte/2018v1/92.pdf

19. Garza-Hernández R, Melendez-Méndez C, Castillo-Martínez G, González-Salinas F, FangHuerta MD, Hidalgo HC. Surgical patients' perception about behaviors of humanized nursing care. Hisp Health Care Int. [Internet]. 2019 [citado em 18 dez 2019]; 18(1):1540415319856326. Disponível em: https://journals.sagepub.com/doi/abs/10.1177/1540415319856326?journalCode=hcia. DOI: https://doi.org/10.1177/1540415319856326

20. Kullberg A, Sharp L, Johansson H, Bergenmar M. Information exchange in oncological inpatient care-Patient satisfaction, participation, and safety. Eur J Oncol Nurs. [Internet]. 2015 [citado em 18 dez 2019]; 19(2):142-7. Disponível em: https://www.cancernurse.eu/documents/EJONInformationExchangeInOncologicalInpatientC arePatientSatisfactionParticipationAndSafety.pdf. DOI: https://doi.org/10.1016/j.ejon.2014.10.005

21. Martins PF, Perroca MG. Satisfação do paciente e acompanhante quanto ao atendimento de necessidades de cuidados de enfermagem. Rev Eletr Enferm. [Internet]. 2017 [citado em $18 \mathrm{dez}$ 2019]; 19:1-11. Disponível em: https://revistas.ufg.br/fen/article/view/41138/23928. DOI: https://doi.org/10.5216/ree.v19.41138

22. Silva N, Gabatz RI, Lemes RA. Percepção do paciente traumatológico acerca da assistência prestada durante a hospitalização. Rev Enferm UFSM [Internet]. 2016 [citado em 8 dez 2019]; 6(3):393-403. Disponível em: https://periodicos.ufsm.br/reufsm/article/view/21306/pdf. DOI: http://dx.doi.org/10.5902/2179769221306

23. Suhonen R, Charalambous A, Berg A, Katajisto J, Lemonidou C, Patiraki E, et al. Hospitalised cancer patients' perceptions of individualised nursing care in four European countries. Eur J Cancer Care [Internet]. 2018 [citado em 8 dez 2019]; 27(1):e12525. Disponível em: https://onlinelibrary.wiley.com/doi/pdf/10.1111/ecc.12525.

https://doi.org/10.1111/ecc.12525

24. Yan J, Liu K, Zhang L, Chu T, Wang X. Patient reporting of undesirable events: a pilot study in China. Int J Qual Health Care [Internet]. 2017 [citado em 8 dez 2019]; 29(3):360-5. Disponível em: https://academic.oup.com/intqhc/article/29/3/360/3065339. DOI: https://doi.org/10.1093/intqhc/mzx029 


\section{Editora Associada: Fernanda Carolina Camargo}

\section{CONTRIBUIÇÕES}

Patricia Nunes Silva participou da concepção, coleta e análise dos dados, redação e revisão. Lúcia Aparecida Ferreira contribuiu na concepção e revisão.

\section{Como citar este artigo (Vancouver)}

Silva PN, Ferreira LA. Percepção dos pacientes sobre a internação hospitalar em diferentes clínicas: uma revisão integrativa. REFACS [Internet]. 2021 [citado em inserir dia, mês e ano de acesso]; 9(Supl. 1):312-322. Disponível em: inserir link de acesso. DOI: inserir link do DOI

\section{Como citar este artigo (ABNT)}

SILVA, P. N.; FERREIRA, L. A. Percepção dos pacientes sobre a internação hospitalar em diferentes clínicas: uma revisão integrativa. REFACS, Uberaba, MG, v. 9, p. 312-322, 2021. Supl. 1. DOI: inserir link do DOI. Disponível em: inserir link de acesso. Acesso em: inserir dia, mês e ano de acesso.

\section{Como citar este artigo (APA)}

Silva, P.N., \& Ferreira, L.A. (2021). Percepção dos pacientes sobre a internação hospitalar em diferentes clínicas: uma revisão integrativa. REFACS, 9(Supl. 1), 312-322. Recuperado em inserir dia, mês e ano de acesso de inserir link de acesso. DOI: inserir link do DOI. 